\title{
Impacto de la educación para el emprendimiento en la creatividad: Una experiencia en Chile con Propensity Score Matching
}

\author{
Impact of Entrepreneurship Education on Creativity: \\ An Experience With Propensity Score Matching in Chile
}

\author{
Francisco Olivos \\ Corporación para el Desarrollo Productivo de Antofagasta, \\ Chile \\ fjolivos@cdp.cl \\ Isabel Álvarez ${ }^{2}$ \\ Corporación para el Desarrollo Productivo de Antofagasta \\ Chile \\ isabelalvarez@cdp.cl \\ Fabián Díaz \\ Corporación para el Desarrollo Productivo de Antofagasta \\ Chile \\ fabiandiaz@cdp.cl
}

Recibido 10 de junio de 2013 • Corregido 11 de julio de 2013 • Aceptado 31 de julio de 2013

Resumen. Este artículo presenta la evaluación del programa Yo aprendo y emprendo ejecutado en la ciudad de Antofagasta, Chile. El programa tiene como objetivo contribuir al desarrollo de jóvenes estudiantes a través de la entrega de herramientas que les permitan incorporar el espíritu emprendedor como actitud de vida y formación. Se realizó una evaluación de impacto mediante Propensity Score Matching, a una muestra de 150 estudiantes de cinco escuelas secundarias. En general, la evaluación sugiere que el programa tiene un efecto positivo sobre el puntaje obtenido por los estudiantes en la prueba de imaginación creativa y las subpruebas de creatividad narrativa y creatividad gráfica.

Palabras claves. Educación, creatividad, emprendimiento, propensity score matching.

1 Máster y licenciado en Sociología de la Pontificia Universidad Católica de Chile. Con estudios en la Universidad de Colonia (Alemania) y en la Pontificia Universidad Católica de San Pablo (Brasil). Actualmente se desempeña como investigador de la Corporación para el Desarrollo Productivo de Antofagasta, Chile y docente de sociología en la Universidad de Antofagasta. Posee artículos publicados y presentaciones en congresos en las áreas de responsabilidad social empresarial.

2 Ingeniera civil y licenciada en Ciencias Básicas de la Universidad Católica del Norte. Diplomada en Administración y Dirección de Proyectos por la Pontificia Universidad Católica de Chile, con cursos de especialización en áreas de sustentabilidad y proyectos. Actualmente se desempeña como jefa del Área de Desarrollo de Programas y Proyectos de la Corporación para el Desarrollo Productivo de Antofagasta, Chile.

3 Candidato a Magister en Psicología Social mención comunitaria, Universidad de Chile. Psicólogo de la Universidad Católica del Norte. Se ha especializado en el desarrollo de programas educacionales y actualmente se desempeña como coordinador de proyectos sociales y de mención comunitaria en la Corporación para el Desarrollo Productivo de Antofagasta. 
URL: http://www.una.ac.cr/educare

CORREO: educare@una.cr

\begin{abstract}
This paper is based on the evaluation of the program Yo Aprendo y Emprendo [I learn and take action] conducted in Antofagasta, Chile. This program aims to contribute to the development of young students providing them with tools to embrace an entrepreneurial culture in their life and formation. An evaluation was conducted through Propensity Score Matching with a sample of 150 students from five secondary schools. In general, the evaluation suggests that the program has a positive effect on the scores obtained by the students in the creative imagination test and in the narrative creativity and graphical creativity subtests.
\end{abstract}

Keywords. Education, creativity, entrepreneurship, propensity score matching.

La creatividad se reconoce como una competencia altamente valorada en las economías de mercado. Al respecto, Schumpeter (1971) reconocía que la "destrucción creativa" era el hecho esencial del capitalismo moderno y el impulso fundamental que lo pone y mantiene en pie. Este proceso procede de la creación de nuevos bienes, de nuevas empresas, de nuevos mercados, de nuevas formas de organización y, en definitiva, de las capacidades innovadoras de la persona emprendedora. Así, la capacidad de creación se transforma en una de las principales competencias de toda empresa de emprendimiento y uno de los desafíos más grandes en la búsqueda de crecimiento económico sostenido a largo plazo.

Distintas instancias políticas e investigaciones empíricas sugieren que es posible aumentar el nivel de emprendimiento con educación (Comisión Europea, 2006). Drucker (1993) argumenta que el emprendimiento no es una magia ni un misterio, sino una disciplina $y$, como tal, puede ser aprendida y enseñada. De igual modo, Kuratko (2005) señala que la visión de un emprendimiento estático es obsoleta, ya que la concepción que debe predominar es la de un emprendimiento que puede ser impulsado a través de la intervención.

De esta forma, se han elaborado distintos paradigmas de "educación emprendedora" a través de la creación de currículos y programas que persiguen fomentar intenciones y competencias del emprendimiento en distintos niveles educacionales. En el universitario se ha mostrado, por ejemplo, que estudiantes participantes en programas de educación emprendedora tuvieron tres veces más probabilidades de iniciar un negocio que quienes no lo hicieron (Charney y Libecap, 2000).

Para el caso de la educación secundaria, una de las experiencias más reconocidas internacionalmente es el Junior Achievement Young Enterprise student mini-company en Estados Unidos y Europa. El programa involucra a jóvenes que asumen responsabilidades como equipo, en un negocio de tamaño pequeño y de corto plazo desde su creación (primeros años de secundaria) hasta liquidación (año final de la secundaria). Sin embargo, la evaluación experimental de Oosterbeek, van Praag y ljsselstein (2010), en un caso concreto de ejecución de este programa, muestra que no tuvo efectos significativos sobre la autoevaluación de capacidades empresariales e, incluso, efectos negativos sobre la motivación para convertirse en un empresario. No obstante, otros resultados han sugerido efectos positivos de programas que buscan impulsar el espíritu emprendedor en las intenciones de emprendimiento (Mueller, 2011; Peterman y Kennedy, 2003; Souitaris, Zerbinati y Al-Laham, 2007; Von Graevenitz, Harhoff y Weber, 2010) y habilidades no cognitivas como la creatividad (Rosendahl, Sloof y Van Praag, 2012). 
A pesar de que existen antecedentes de programas de educación para el emprendimiento desde hace 45 años (Kuratko, 2005), se reconoce una brecha de conocimiento entre el desarrollo de esos programas y evaluaciones de impacto en Estados Unidos y Europa (Oosterbeek et al, 2010). Esta situación no es diferente para Latinoamérica, dado que existen programas de formación de emprendedores, pero no existen políticas de evaluación de impacto experimental o cuasi-experimental de estos programas en la incorporación de los jóvenes al mercado laboral, creación de empresas o el mejoramiento de habilidades cognitivas o no cognitivas.

El caso más importante de evaluación de este tipo de programas ha sido realizado en Colombia (Varela y Jiménez, 2001). Los investigadores buscaron evaluar el efecto en la decisión de convertirse en emprendedores para alumnos de dos universidades del país. Al momento del estudio, entre un $23 \%$ y $7 \%$ de los encuestados trabajaba en su propio negocio. No obstante el aporte de esta evidencia, no se utilizan metodologías experimentales o cuasi-experimentales que puedan evitar sesgos como el de la auto-selección, por lo cual sigue siendo necesario avanzar en este conocimiento para el mejoramiento de los programas de emprendimiento en Latinoamérica.

En este sentido, en este artículo se realiza una evaluación de impacto cuasi-experimental del programa Yo Aprendo y emprendo ejecutado en la ciudad de Antofagasta, Chile. El programa tiene como objetivo contribuir al desarrollo de jóvenes estudiantes a través de la entrega de herramientas que les permitan incorporar la cultura del emprendimiento como actitud de vida y formación. Los resultados sugieren que existe un efecto positivo del programa de educación para el emprendimiento en la creatividad general y gráfica de los estudiantes beneficiarios, en comparación a los no beneficiarios.

Por otra parte, el programa es financiado por el sector privado y constituye también un avance en el vacío reconocido en la bibliografía en cuanto a la ausencia de evaluaciones de impacto de actividades de responsabilidad social empresarial (Salazar, Husted y Biehl 2012). En este sentido, este artículo es un prospecto para el mejoramiento de las intervenciones privadas.

El artículo se organiza en 4 secciones: se comienza con una descripción del programa evaluado y su contexto, para luego especificar el diseño metodológico de la evaluación en cuanto a las características de la muestra, instrumentos, modelos estadísticos, balanceo y limitaciones. En una tercera sección se reportan los resultados de la evaluación y, finalmente, se agregan algunas conclusiones en cuanto a la experiencia de educación para el emprendimiento en Latinoamérica.

\section{El programa Yo aprendo y emprendo}

Yo aprendo y emprendo surge a partir de una iniciativa privada para la implementación de un programa de emprendimiento para las diversas unidades educacionales públicas. Su objetivo es concientizar a jóvenes estudiantes de enseñanza media de la comuna de Antofagasta, Chile, respecto a las competencias mayormente vinculadas con el emprendimiento. Posteriormente, se inicia un trabajo en la creación y ejecución de un plan de intervención, orientado al fortalecimiento del aprendizaje respecto al emprendimiento, por medio del desarrollo de capacidades centrales en el ámbito creativo, 
URL: http://www.una.ac.cr/educare

CORREO: educare@una.cr

trabajo en equipo, autoconocimiento y comunicación. La intervención tiene una duración de tres años, desde segundo año de secundaria y hasta cuarto año, donde se finaliza esta etapa educacional. Aquí se presenta la evaluación de impacto del primer año de programa ejecutado hasta la fecha.

Existe evidencia para el caso de estudiantes de educación superior en la ciudad de Antofagasta que demuestra que un currículo orientado hacia la creación de empresas y al fortalecimiento del espíritu emprendedor contribuye a la propensión de creación de empresas (Romaní, Didonet, Contuliano y Portilla, 2013). Esto sugiere la posibilidad de generar intervenciones a través del currículo para incentivar ciertas actitudes emprendedoras. Sin embargo, se carece de estudios similares que lo demuestren en niveles educacionales menores.

Como se presenta en la Figura 1, la intervención se realizó en 3 etapas: Comprender el emprender, Talleres de comunicación y debates y Desarrollo de ideas. La población objetivo corresponde a estudiantes de educación secundaria de establecimientos de dependencia municipal en la comuna de Antofagasta, Chile. Se designan, como los establecimientos beneficiarios, los 5 liceos de dependencia municipal ${ }^{4}$ más vulnerables de la ciudad, medida por el porcentaje de alumnos con familia bajo la línea de la pobreza. Del total de establecimientos, la población total de estudiantes beneficiarios del programa fue de 150, de los cuales 135 finalizaron el programa con un 10\% de atrición. La decisión de participar en el programa fue voluntaria, para lo cual se realizó un trabajo de difusión por parte de los monitores y profesores para la incorporación de estudiantes como beneficiarios. Este hecho particular es la principal limitante para una evaluación experimental con asignación aleatoria, por lo cual se han utilizado herramientas estadísticas para su solución.

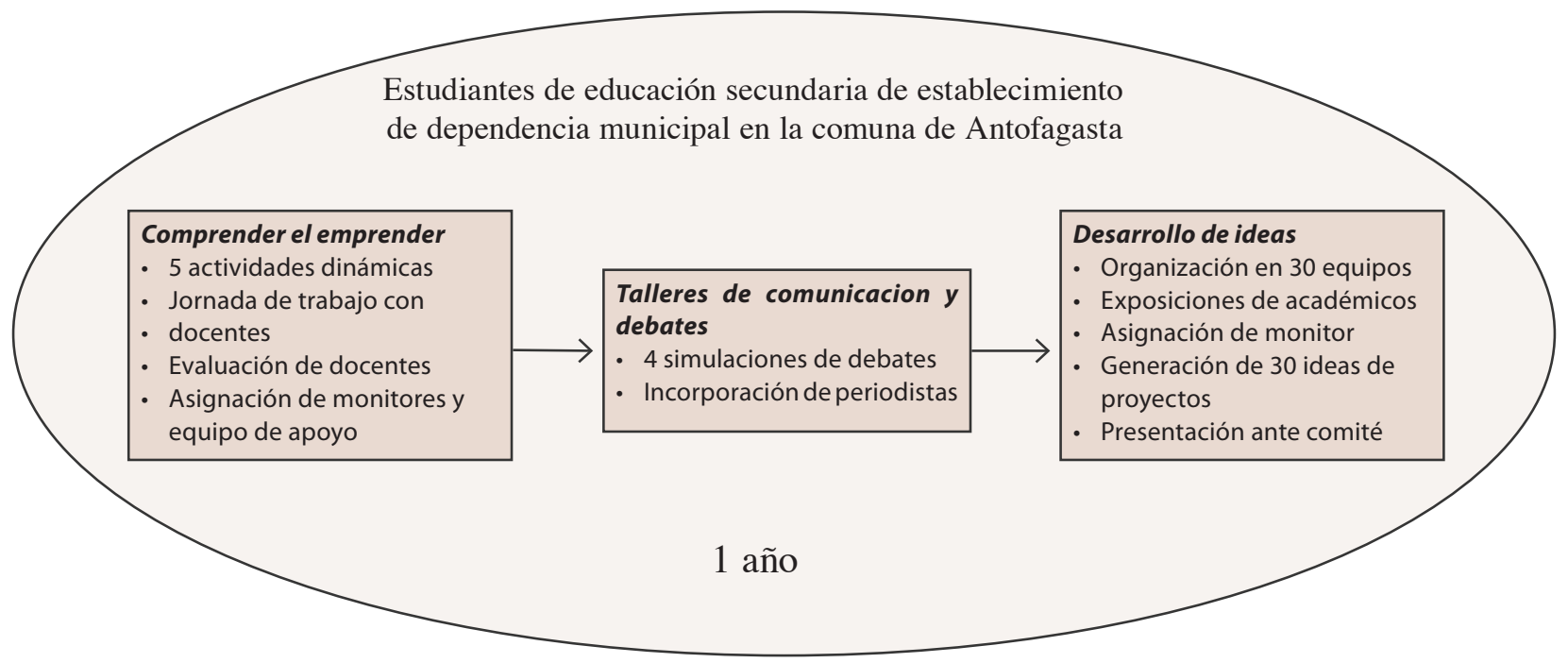

Figura 1. Etapas y actividades del programa Yo aprendo y emprendo. Elaboración propia.

4 En Chile existen tres tipos de establecimientos educacionales por dependencia: establecimientos municipales dependientes del gobierno local, establecimientos subvencionados por el Estado y establecimientos particulares. Para una descripción del modelo educacional chileno ver Cox (2005). 
Se diseñaron una serie de actividades y dinámicas, en las cuales se combinan el conocimiento aplicable y la práctica, con el fin de ahondar entre las diversas habilidades potenciadoras de la acción de emprender. Se busca concientizar al estudiantado, respecto a la forma de aplicar el concepto de emprender en los distintos círculos sociales que lo involucran como ciudadano, para que por sí mismos logren visualizar y sensibilizarse ante las múltiples oportunidades y beneficios que poseen, de manera que integren, en su diario vivir, un pensamiento divergente y lateral. La intervención está compuesta por tres etapas que se describen a continuación.

La primera parte de la intervención se denominó Comprender el emprender. En esta se efectuaron actividades de tipo dinámicas que buscaban fortalecer la creatividad de sus participantes, basándose en los conceptos teóricos asociados a la fluidez, flexibilidad, originalidad y elaboración (etapas del proceso creativo); además de los lineamientos planteados en la estructura de pensamiento de diseño -asociados a los procesos de inspirarse, idear, implementar y testear-, persiguiendo, paralelamente, la concientización respecto a las competencias de mayor vinculación con el emprendimiento. En cada sesión de 4 horas de duración, ejecutadas directamente en dependencias de cada establecimiento educacional, se realizaron 5 actividades. Estas fueron consignadas en un orden secuencial de dificultad, lo cual permitió a los participantes generar procesos de adaptación sobre las estrategias pedagógicas implementadas (el contenido y las capacidades fortalecidas en cada actividades se presentan en el material complementario).

Con tal de fomentar un mayor involucramiento del cuerpo docente participante en el proyecto, además de potenciar conceptos técnicos vinculados con el proceso de emprender, se instauró una primera jornada de trabajo de 8 horas, donde se plantearon las líneas de acción que estos últimos debían seguir para potenciar el proceso de aprendizaje del alumnado en el trabajo de aula.

Posteriormente, dentro del cuarto taller de la etapa 1, se ejecutó un proceso de evaluación práctica del personal docente participante, instancia denominada "Profesores en Acción", que permitió verificar el nivel de comprensión, integración e implementación de las herramientas pedagógicas transferidas, además de observar el nivel de compromiso e involucramiento con el desarrollo del proyecto. En esta actividad, el personal docente debía diseñar una actividad que potenciara las habilidades de creatividad e innovación en el estudiantado, basándose en los argumentos técnicos trasmitidos durante el taller inicial y las posteriores actividades ejecutadas en el aula.

Con tal de responder de forma adecuada a las disponibilidades horarias consignadas por cada establecimiento, se decidió designar una persona monitora por institución, para así facilitar su gestión, en el caso de la existencia de cambios en las programaciones iniciales organizadas entre el equipo de organización externa ejecutora y las unidades pedagógicas correspondientes. Cada monitor fue rigurosamente seleccionado, con base en su pertinencia profesional y de experiencia en el trabajo educacional con adolescentes.

Además, se consignó un equipo de apoyo y asesoramiento técnico, quienes se encargaron de velar por el correcto funcionamiento operativo y la congruencia metodológica entre las 
URL: http://www.una.ac.cr/educare

CORREO: educare@una.cr

diversas actividades paralelas realizadas en cada establecimiento. Este equipo de soporte se responsabiliza de que exista un alto grado de similitud entre las actividades ejecutadas en diversas fechas distintas, con tal de no ver diferenciado el proceso educativo del estudiantado por factores ambientales, de infraestructura o externos a la intervención.

En la segunda etapa de la intervención, denominada Talleres de comunicación y debates, se desarrollaron actividades de tipo dinámicas, en busca, principalmente, del fortalecimiento de las habilidades interpersonales orientadas a la acción de debatir, comprendidas como la comunicación efectiva, persuasión, negociación, manejo de la presión, entre otras. A su vez, se trasmitieron herramientas vinculadas con la construcción de argumentos. Este proceso se reforzó con la supervisión y participación de profesionales periodistas.

Para esta etapa, se configuró un cronograma de acción con base en las 4 temáticas definidas para el proyecto, las cuales fueron: desarrollo social, eficiencia energética y recursos hídricos, reciclaje e innovación en recursos naturales. En cada sesión de 4 horas de duración, se realizaron 4 actividades, consignadas en un orden secuencial de dificultad. Esto permitió a sus participantes, inicialmente, obtener las herramientas necesarias para la construcción de argumentos, para luego ponerlos en práctica mediante situaciones simuladas de discusión y debate.

Para el segundo taller de la presente etapa se consideró la participación de profesionales periodistas para asesorar al estudiantado en la actividad de simulación de un debate, donde se debía actuar como abogados acusadores o defensores dentro de una situación planteada directamente asociada a cada temática específica abarcado por el taller.

Finalmente, en la tercera etapa denominada Desarrollo de ideas, se dividió al estudiantado en 30 equipos de trabajo. El objetivo fue participar en un concurso a partir de la generación de una idea de proyecto, que se enmarcó en los siguientes temas: desarrollo social, reciclaje de residuos, innovación en recursos naturales, y eficiencia en energía y en recursos hídricos. Posteriormente, presentaron su proyecto ante una comisión evaluadora al finalizar la intervención durante el año 2012.

Con la finalidad de que los estudiantes fueran orientados técnicamente en la ejecución de la idea de proyecto, cada establecimiento contó con una persona monitora a cargo, quien trabajó semanalmente durante tres meses con los equipos y con su profesor o profesora. Se apoyó a los estudiantes con 5 charlas informativas realizadas por personal académico de una universidad de la región.

\section{El diseño del cuasi-experimento}

\section{Propensity score matching}

Los modelos clásicos para evaluar programas de intervención buscan responder cuál hubiese sido el resultado para el tratado, si este no hubiese participado en el programa, lo que se define como el contrafactual. Para el programa la pregunta es cuál hubiese sido el efecto en los estudiantes del programa Yo aprendo y emprendo, si estos no hubiesen participado en él. Si 
definimos $Y_{\mathrm{i} 1}$ para el outcome del participante (grupo de tratamiento) y $Y_{\mathrm{i} 0}$ para el no participante (grupo de control), el efecto esperado del tratamiento sería:

$$
\Delta=E\left(Y_{\mathrm{i} 1}\right)-E\left(Y_{\mathrm{i} 0}\right) .
$$

Definiendo $T=1$ para los participantes y $T=0$ para los no participantes:

$$
\left.\Delta\right|_{\mathrm{t}=1}=E\left(Y_{\mathrm{i} 1} \mid T_{\mathrm{i}}=1\right)-E\left(Y_{\mathrm{i} 0} \mid T_{\mathrm{i}}=1\right) \text {. }
$$

Sin embargo, los datos no experimentales presentan problemas de autoselección de los beneficiarios, lo cual genera que se obtengan estimadores sesgados del efecto del programa. En el caso de un diseño experimental, el sesgo desaparece porque existe una selección aleatoria de participantes y no participantes que balancea las características inobservadas de los individuos. En el caso de la segunda ecuación, el efecto sería insesgado. Al hablar de selección aleatoria, se está haciendo referencia a que $Y_{\mathrm{i} 1}, Y_{\mathrm{i} 0} \perp T_{\mathrm{i},}$ donde $\perp$ indica independencia. Así, la distribución condicional de las características es igual para participantes y no participantes. En este caso $E\left(Y_{\mathrm{i} 1} \mid T_{\mathrm{i}}=1\right)=E\left(Y_{\mathrm{i} 0} \mid T_{\mathrm{i}}=0\right)$, por lo tanto:

$$
\left.\Delta\right|_{\mathrm{t}=1}=E\left(Y_{\mathrm{i} 1} \mid T_{\mathrm{i}}=1\right)-E\left(Y_{\mathrm{i} 0} \mid T_{\mathrm{i}}=1\right)=E\left(Y_{\mathrm{i} 1} \mid T_{\mathrm{i}}=1\right)-E\left(Y_{\mathrm{i} 0} \mid T_{\mathrm{i}}=0\right)
$$

En el caso de datos no experimentales no se cuenta con la información para $E\left(Y_{i 0} \mid T_{i}=0\right)$ y una de las soluciones posibles es recurrir a un diseño no experimental con base en Propensity Score Matching (PSM), estimador no paramétrico que permite solucionar el problema de falta de información, al generar una muestra de individuos comparables con los participantes del programa, según ciertas características observables. De acuerdo con Rosenbaum y Rubin (1983), para definir la probabilidad condicional de participar en un programa se asigna un vector de covariantes observadas $X$. Entonces, $\mathrm{P}(\mathrm{X})=\operatorname{prob}(T=1 \mid X)=\mathrm{E}(\mathrm{W} \mid \mathrm{X})$.

El vector elimina las diferencias entre el grupo de control y de tratamiento, con lo cual permite la independencia condicional $\mathrm{E}\left(\mathrm{Y}_{\mathrm{i} 0} \mid \mathrm{T}_{\mathrm{i}}=1, \mathrm{P}(X)\right)=\mathrm{E}\left(\mathrm{Y}_{\mathrm{i} 0} \mid \mathrm{T}_{\mathrm{i}}=0, \mathrm{P}(X)\right)$. Por lo tanto:

$$
\begin{gathered}
\left.\Delta\right|_{\mathrm{t}=1}=E\left(Y_{\mathrm{i} 1} \mid T_{\mathrm{i}}=1, \mathrm{P}(\mathrm{X})\right)-E\left(Y_{\mathrm{i} 0} \mid T_{\mathrm{i}}=1, \mathrm{P}(\mathrm{X})\right) \\
=E\left(Y_{\mathrm{i} 1} \mid T_{\mathrm{i}}=1, \mathrm{P}(\mathrm{X})\right)-\mathrm{E}\left(\mathrm{Y}_{\mathrm{i} 0} \mid \mathrm{T}_{\mathrm{i}}=0, \mathrm{P}(X)\right)
\end{gathered}
$$

De esta forma, el PSM provee una estimación no sesgada del efecto tratamiento esperado con base en un conjunto de covariantes $X$ observables, y asume que no existen diferencias no observables anteriores al tratamiento entre los participantes y no participantes. Existen diferentes técnicas para la generación de este grupo de control o emparejamiento entre 
URL: http://www.una.ac.cr/educare

CORREO: educare@una.cr

individuos similares. Para este caso, se utiliza le técnica del "vecino más cercano" en donde se realiza el emparejamiento uno-a-uno entre los sujetos del grupo de control y los sujetos del grupo de tratamiento.

\section{Muestra y datos}

Se realizó un muestreo no probabilístico para la selección de tratados y grupo de control. Este rasgo es reconocido por la bibliografía (Rosenbaum, 2002) como una de las principales limitantes en la evaluación de impacto de programas, por las dificultades de asignar el tratamiento aleatoriamente. La muestra inicial antes del PSM cuenta con 150 observaciones, lo que corresponde a la cantidad de individuos con observaciones tanto en la línea base como en la medición post tratamiento. La muestra final de observaciones de individuos comparables después de la realización del PSM es de 132 casos.

Las covariantes observadas escogidas para la realización del emparejamiento fueron el sexo, establecimiento educacional, condición laboral del estudiante, condición laboral del padre y madre, si habita con sus padres, condición de reprobación de algún nivel educacional y estudios superiores de los padres. La estadística descriptiva de estas variables es presentada en la sección de resultados y la propiedad de balanceo se evalúa a continuación.

\section{Propiedad de balanceo}

Uno de los requerimientos para realizar un emparejamiento de forma adecuada es que las covariantes tanto en el grupo de control como de tratamiento se encuentren balanceadas. La tabla 1 presenta las estimaciones de modelos de regresión logística para evaluar el balance de la muestra a partir del PSM. La primera columna muestra el modelo de regresión para la muestra total del estudio y es posible decir a partir de los datos que la probabilidad de ser asignado al tratamiento depende de ciertas características de los estudiantes como el sexo, si vive con los padres y la condición laboral del padre. De esta forma, si fuese utilizada esta muestra para la evaluación, existirían posibles sesgos de selección. Sin embargo, como se presenta en la siguiente columna, y tras la estimación del PSM, la probabilidad de ser beneficiario del programa no estaría afectada por ninguna de las variables condicionales, las que se distribuirían homogéneamente entre el grupo de tratados y de control. Por ejemplo, en el modelo 1, el ser hombre reduce las chances de ser tratado en un 53\% en comparación a ser mujer, diferencia que no es estadísticamente significativa si se realiza el análisis posterior al PSM. Lo mismo sucede con la variable que indica si el estudiante vive con los padres y la condición laboral del padre.

Es importante señalar que no se incluye la variable de educación superior de la madre porque Chile presenta los niveles más altos de homogamia educacional (Torche, 2010) y, por lo tanto, esta variable presenta alta correlación con la educación del padre introduciendo multicolinealidad a los modelos. 
Tabla 1

Regresiones logísticas para prueba de balanceo

\begin{tabular}{|c|c|c|}
\hline & \multicolumn{2}{|c|}{ Odds ratio } \\
\hline & Total & PSM \\
\hline Variable dependiente: Tratamiento & Modelo 1 & Modelo 2 \\
\hline \multirow[t]{2}{*}{ Hombre $^{a}$} & $0.472^{* *}$ & 0.786 \\
\hline & $(0.179)$ & $(0.325)$ \\
\hline \multirow[t]{2}{*}{15 años $^{b}$} & 0.431 & 1.144 \\
\hline & $(0.508)$ & $(1.717)$ \\
\hline \multirow[t]{2}{*}{16 años $^{b}$} & 0.323 & 0.988 \\
\hline & $(0.444)$ & (1.633) \\
\hline \multirow[t]{2}{*}{17 años $^{b}$} & 0.0752 & 0.244 \\
\hline & $(0.119)$ & $(0.444)$ \\
\hline \multirow[t]{2}{*}{ Trabajac $^{c}$} & 0.327 & 0.414 \\
\hline & $(0.226)$ & $(0.281)$ \\
\hline \multirow[t]{2}{*}{ Repitió algún curso ${ }^{d}$} & 0.688 & 0.805 \\
\hline & $(0.512)$ & $(0.596)$ \\
\hline \multirow[t]{2}{*}{ Vive con los padres ${ }^{\mathrm{e}}$} & $2.089 * *$ & 1.509 \\
\hline & $(0.784)$ & $(0.604)$ \\
\hline \multirow[t]{2}{*}{ Padre con estudios superiores ${ }^{f}$} & 1.085 & 0.905 \\
\hline & $(0.621)$ & $(0.618)$ \\
\hline \multirow[t]{2}{*}{ Padre con trabajo ${ }^{g}$} & $2.850 * *$ & 1.925 \\
\hline & $(1.391)$ & $(0.954)$ \\
\hline \multirow[t]{2}{*}{ Madre con trabajo ${ }^{\text {h }}$} & 0.838 & 0.886 \\
\hline & $(0.309)$ & $(0.340)$ \\
\hline \multirow[t]{2}{*}{ Constante } & 2.071 & 0.723 \\
\hline & $(2.784)$ & (1.193) \\
\hline Observaciones & 158 & 132 \\
\hline
\end{tabular}

Nota: Error estándar entre paréntesis. ${ }^{* *} p<0.01,{ }^{* *} p<0.05,{ }^{*} p<0.1$. Categorías de referencia: aMujeres, b14 años, 'No trabaja, dNo ha repetido, eNo vive con los padres, fPadre sin estudios superiores, gPadre sin trabajo y ${ }^{\mathrm{h}}$ Madre sin trabajo. 
URL: http://www.una.ac.cr/educare

CORREO: educare@una.cr

\section{Variable dependiente: Prueba de imaginación creativa}

Para el establecimiento de puntajes iniciales y de impacto, se utilizó la Prueba de imaginación creativa para jóvenes (PIC-J), un instrumento que nos permite evaluar específicamente tanto la creatividad narrativa o verbal como la creatividad figurativa o gráfica. Dentro de las variables del proceso creativo medidas en el PIC-J, se incluyen la fluidez, flexibilidad, originalidad y elaboración asociada a la creatividad narrativa y la originalidad, elaboración, título y detalles especiales dentro de la creatividad gráfica o figurativa. Tanto la creatividad narrativa como gráfica funcionan bajo el prisma de conglomerado de sub áreas, sus puntajes son el resultado de la incorporación de las variables subyacentes señaladas (fluidez, flexibilidad, elaboración, etc.).

Esta prueba está conformada por 4 juegos:

En el juego 1 se le presenta un dibujo al estudiante en el que aparece un niño abriendo un cofre y debe escribir, a partir de este estímulo, todo lo que cree que pueda estar ocurriendo. Esto permite medir la capacidad de formulación de hipótesis e idear las posibles alternativas de acción sobre un contexto determinado, al plantear distintas posibilidades de sucesos respecto a lo que acontece en la escena. Debido a la naturaleza de este proceso, se puede medir en esta instancia la fluidez y flexibilidad del joven a escala narrativa.

Respecto al juego 2, se les pregunta a los niños sobre los usos posibles que puede tener un tubo de goma. Así, se evalúa la capacidad de los individuos para pensar de forma no convencional. Este juego entrega información acerca de la forma de encontrar usos, funciones y aplicaciones distintas de lo habitual, y permite medir la fluidez, flexibilidad y originalidad a escala narrativa.

En el juego 3, al igual que en el 2, favorece el acceso a la capacidad del estudiante para fantasear y manejar ideas poco convencionales, y permite recolectar información acerca de su capacidad de apertura y receptividad ante situaciones novedosas, de manera que se puede medir fluidez, flexibilidad y originalidad a escala narrativa. A los estudiantes se le plantea una situación inverosímil a la que deben dar respuesta: "Imagínate qué ocurriría si cada ardilla, de repente, se convirtiera en un dinosaurio".

Por último, el juego 4 aproxima a conocer la capacidad estudiantil de entregar gráficamente respuestas originales, y proporciona información para discriminar a los individuos elaboradores de productos creativos. Este juego aporta información respecto a la originalidad y elaboración a escala gráfica del individuo, incluyendo variables asociadas al título señalado del dibujo y los detalles especiales de este. Se procede pidiéndoles a los estudiantes que, a partir de unos trazos dados, completen un dibujo y le pongan un título a cada uno. De las figuras incompletas se seleccionan 4 por una submuestra de sujetos considerados creativos, las que son finalmente evaluadas. 
Los criterios de selección para la utilización de este instrumento en el presente estudio se asocian, principalmente, a dos factores claves: El primero, asociado a la incorporación en esta prueba de los 4 factores principales definidos por Guilford (1967) para el pensamiento creativo (fluidez, flexibilidad, originalidad y elaboración), elementos que son sustento teórico clave en el modelo de intervención llevado a cabo en terreno. El segundo lugar, existe bibliografía (Artola y Barraca, 2004) que, en el estudio de proceso de cambios en el pensamiento divergente a lo largo del ciclo vital, ha utilizado directamente la herramienta PIC en sus diversas versiones para la medición de las variaciones observadas entre grupos de niños, jóvenes y adultos en este tipo de capacidades. Asimismo, Artola y Barraca (2004) presentan evidencia de su validez de constructo, validez discriminante y fiabilidad.

Cabe destacar que no existen estudios ni validaciones de este tipo en Latinoamérica para las presentes herramientas psicotécnicas. Al respecto, es la población española la de mayor similitud a nivel estadístico.

\section{Limitantes del estudio}

Una de las principales limitantes metodológicas del estudio es que existen riesgos de contaminación de la muestra de control. Principalmente porque los profesores capacitados para realizar los talleres reconocen haber aplicado en las aulas los conocimientos y experiencias adquiridas en los talleres de capacitación del programa. Sin embargo, esto causaría una subestimación del efecto, por lo que, si se identifica un efecto positivo, este debería seguir existiendo en un escenario de independencia de las muestras. Idealmente, se debió haber generado una muestra de control a partir de estudiantes de otros establecimientos educacionales.

Por otra parte, el método de PSM no soluciona el problema de sesgo de selección en donde la probabilidad de ser tratado puede estar siendo explicada por variables no observadas. El efecto de estas variables no observadas sobre el outcome puede ser distinto para los individuos de la muestra de tratamiento y de la de control. Por ejemplo, se podría pensar en que la participación en el programa es voluntaria y esta voluntariedad podría estar motivada por una cierta inclinación hacia prácticas de la creatividad que estarían presentes en un menor grado en aquellos que no decidieron participar y forman parte de la muestra de control. Así, el cambio en el outcome podría estar explicado por esta diferencia y no necesariamente por el tratamiento.

En este sentido, una de las recomendaciones para hacer frente a este problema es el análisis de sensibilidad propuesto por Rosembaum (2002), que evalúa en qué medida estas variables no observadas alteran la inferencia sobre los efectos del programa. Para el presente estudio se desconoce la probabilidad de que dos individuos con las mismas características sean asignados al tratamiento, definida como Г. Por lo tanto, se realiza un test en donde se observa si varían las conclusiones del estudio para variaciones marginales de $\Gamma$ que reflejan el sesgo de variables no observadas. Los estudios experimentales tienen un $\Gamma=1$, en donde no existen 
URL: http://www.una.ac.cr/educare

CORREO: educare@una.cr

diferencias de probabilidades de ser asignados al grupo de tratamiento, en cambio en al caso donde $\Gamma=2$ las probabilidades aumentan al doble.

Como se puede observar en la tabla 2, el análisis de sensibilidad mediante Rosenbaum Bounds sugiere que la significancia del efecto identificado sería insensible a un sesgo de selección. La conclusión general que se obtiene de este test es que el efecto tratamiento del programa Yo aprendo y emprendo es insensible a posibles sesgos de variables no observadas. Las conclusiones para la prueba de imaginación creativa dejarían de ser significativas solo si las probabilidades de ser tratados cuadriplicarían a las de no serlo, y esto solo a un nivel de confianza del $90 \%$.

Tabla 2

Análisis de sensibilidad

\begin{tabular}{ccc}
\hline$\Gamma$ & Sig $^{+}$ & Sig \\
\hline 1.0 & 0.000 & 0.000 \\
1.5 & 0.000 & 0.000 \\
2.0 & 0.000 & 0.000 \\
2.5 & 0.000 & 0.000 \\
3.0 & 0.002 & 0.000 \\
3.5 & 0.006 & 0.000 \\
4.0 & 0.012 & 0.000 \\
\hline
\end{tabular}

Los análisis de sensibilidad son el método más robusto desarrollado en la actualidad para poder identificar posibles sesgos de selección, y la evidencia de este estudio sugiere que las conclusiones posiblemente estén libres del sesgo que podrían introducir variables no observadas.

\section{Resultados}

En la tabla 3 se presenta la distribución de las características sociodemográficas de la muestra de la línea base, tanto para los beneficiados del programa como para el grupo de control. Asimismo, se presenta su distribución final luego de la estimación del PSM. Las variables que se incluyen en la tabla 3 son aquellas que se utilizaron como covariantes en el PSM. 
Tabla 3

Características sociodemográficas de la muestra (\%)

\begin{tabular}{|c|c|c|c|c|c|c|}
\hline & \multicolumn{3}{|c|}{ Muestra total } & \multicolumn{3}{|c|}{ PSM } \\
\hline & Control & Tratamiento & Total & Control & Tratamiento & Total \\
\hline \multicolumn{7}{|l|}{ Sexo } \\
\hline Mujer & 33 & 48 & 42 & 36 & 36 & 36 \\
\hline Hombre & 67 & 52 & 58 & 64 & 64 & 64 \\
\hline Total & 100 & 100 & 100 & 100 & 100 & 100 \\
\hline \multicolumn{7}{|l|}{ Establecimiento } \\
\hline 1 & 19 & 25 & 22 & 20 & 27 & 23 \\
\hline 2 & 17 & 15 & 16 & 17 & 14 & 15 \\
\hline 3 & 22 & 22 & 22 & 23 & 18 & 20 \\
\hline 4 & 22 & 16 & 18 & 21 & 17 & 19 \\
\hline 5 & 20 & 22 & 22 & 20 & 24 & 22 \\
\hline Total & 100 & 100 & 100 & 100 & 100 & 100 \\
\hline \multicolumn{7}{|l|}{ Edad } \\
\hline 14 años & 1 & 4 & 3 & 2 & 2 & 2 \\
\hline 15 años & 57 & 72 & 65 & 56 & 68 & 62 \\
\hline 16 años & 29 & 21 & 25 & 29 & 27 & 28 \\
\hline 17 años & 13 & 2 & 7 & 14 & 3 & 8 \\
\hline Total & 100 & 100 & 100 & 100 & 100 & 100 \\
\hline
\end{tabular}

Trabaja

\begin{tabular}{|c|c|c|c|c|c|c|}
\hline $\mathrm{Nc}$ & 6 & 96 & 91 & 85 & 94 & 89 \\
\hline Sí & 14 & 4 & 9 & 15 & 6 & 11 \\
\hline Total & 100 & 100 & 100 & 100 & 100 & 100 \\
\hline
\end{tabular}

Repitió algún nivel

\begin{tabular}{rrrrrrr} 
No & 55 & 75 & 66 & 55 & 68 & 61 \\
\multicolumn{1}{c}{ Sí } & 45 & 25 & 34 & 45 & 32 & 39 \\
\hline Total & 100 & 100 & 100 & 100 & 100 & 100 \\
\hline
\end{tabular}

Vive con padres

\begin{tabular}{|c|c|c|c|c|c|c|}
\hline No & 52 & 37 & 44 & 55 & 48 & 52 \\
\hline Sí & 48 & 63 & 56 & 45 & 52 & 48 \\
\hline Total & 100 & 100 & 100 & 100 & 100 & 100 \\
\hline
\end{tabular}

Padre con estudios superiores

\begin{tabular}{|c|c|c|c|c|c|c|}
\hline $\mathrm{N}$ & 87 & 89 & 88 & 89 & 92 & 91 \\
\hline Sí & 13 & 11 & 12 & 11 & 8 & 9 \\
\hline Total & 100 & 100 & 100 & & & \\
\hline
\end{tabular}


URL: http://www.una.ac.cr/educare

CORREO: educare@una.cr

\begin{tabular}{lrrrrrr}
\hline & \multicolumn{3}{c}{ Muestra total } & & PSM \\
\hline & Control & Tratamiento & Total & Control & Tratamiento & Total \\
\hline $\begin{array}{l}\text { Padre con trabajo } \\
\quad \text { No }\end{array}$ & 28 & 10 & 18 & 24 & 14 & 19 \\
$\quad$ Sí & 72 & 90 & 82 & 76 & 86 & 81 \\
\hline Total & 100 & 100 & 100 & 100 & 100 & 100 \\
\hline Madre con trabajo & & & & & & \\
$\quad$ No & 48 & 53 & 51 & 48 & 53 & 51 \\
$\quad$ Sí & 52 & 47 & 49 & 52 & 47 & 49 \\
\hline Total & 100 & 100 & 100 & 100 & 100 & 100 \\
\hline
\end{tabular}

Como se observa, para la muestra total existe una mayoría de hombres (58\%), predominancia de estudiantes con 15 años de edad (65\%), que no trabajan (91\%), no han repetido ningún nivel (66\%), que viven con sus padres (56\%), de los cuales la mayoría de los papás no tienen estudios superiores (88\%) y trabajan (82\%), al igual que las madres en este último aspecto (51\%). Si bien las magnitudes cambian al realizar el análisis de tratados y no tratados, las tendencias se mantienen.

El objetivo de este estudio es medir el impacto que tuvo el programa Yo aprendo y emprendo en los estudiantes participantes de la iniciativa. Para esto se estiman "diferencias en diferencias" tanto para el puntaje general de la Prueba de imaginación creativa como de las subpruebas de Creatividad gráfica y creatividad narrativa. Esto se resume en la tabla 4.

Los resultados indican que existen efectos de la participación en el programa para las tres pruebas. Como se observa para la PIC-J, el aumento de 54 puntos para el grupo de tratamiento difiere significativamente $(p=0.02)$ de la diferencia entre el puntaje de la prueba antes y después del tratamiento para el grupo de control. La diferencia de 15 puntos entre el aumento para el grupo de control y el aumento para el grupo de tratamiento se sugieren como el efecto promedio de participar en el programa.

Por otra parte, el efecto también se identifica para las subpruebas de creatividad gráfica y creatividad narrativa. Existe una diferencia entre la variación para el grupo de tratamiento y para el grupo de control de 4 puntos en el caso de la creatividad gráfica y de 10,7 puntos en la creatividad narrativa, ambas diferencias estadísticamente significativas ( $p=0.00$ y $p=0.08$ respectivamente).

Tabla 4

Diferencia de diferencias por pruebas

\begin{tabular}{lccccccc}
\hline & \multicolumn{3}{c}{ Grupo de tratamiento $(\mathrm{n}=66)$} & \multicolumn{2}{c}{ Grupo de control $(\mathrm{n}=66)$} & Test de diferencia \\
\hline & $\mathrm{T} 0$ & $\mathrm{~T} 1$ & Diferencia & $\mathrm{T} 0$ & $\mathrm{~T} 1$ & Diferencia & $\mathrm{p}>|\mathrm{t}|$ \\
\hline PIC-J & 78 & 132 & 54 & 40 & 79 & 39 & 0.02 \\
Creatividad gráfica & 7.8 & 13.2 & 5.4 & 6.8 & 8.2 & 1.4 & 0.00 \\
Creatividad narrativa & 70.7 & 119.0 & 48.3 & 33.4 & 71.0 & 37.6 & 0.08 \\
\hline
\end{tabular}


En resumen, la estimación del PSM permite identificar efectos positivos de la participación en el programa. Específicamente se logró identificar un impacto en el puntaje general de imaginación creativa y en la subprueba de creatividad gráfica, no así en la prueba de creatividad narrativa. Esta evidencia muestra que el programa Yo aprendo y emprendo ha logrado constituirse un una iniciativa útil en la generación y fomento del espíritu emprendedor.

\section{Conclusión}

La creatividad es uno de las capacidades más importantes en momentos en donde la innovación, la tecnología y el emprendimiento son destacados como los rasgos más importantes de nuestra sociedad. Aquí se ha logrado presentar cómo es posible impulsar la creatividad y, en definitiva, el nivel de emprendimiento a través de la educación o de intervenciones sistemáticas en contextos educacionales.

Las iniciativas de impulso al emprendimiento se realizan recurrentemente en la educación universitaria. Sin embargo, la evidencia aquí presentada muestra que es posible intervenir en niveles educacionales menores, fomentando el espíritu emprendedor desde etapas en donde el pensamiento abstracto y la incorporación de normas sociales se encuentran en pleno desarrollo. Este es uno de los principales elementos del diseño del programa Yo aprendo y emprendo que lo diferencia con otras iniciativas a nivel internacional como es el caso del Junior Achievement Young Enterprise student mini-company, que se focaliza en estudiantes post-secundarios y, que como se ha citado (Oosterbeek et al (2010), no presenta efectos significativos e incluso negativos. El efecto de las intervenciones de currículo como sugieren Romaní et al. (2013) para el caso de estudiantes universitarios, podría ser replicado a partir de lo expuesto en estudiantes de educación secundaria.

Por lo tanto, el programa Yo aprendo y emprendo se transforma en un modelo de intervención que ha mostrado tener impactos positivos en el nivel de creatividad de los beneficiarios. De esta forma, se destaca la importancia de incluir evaluaciones de impacto en intervenciones educacionales, ya que solo de esta forma es posible argumentar con evidencia el éxito de una intervención y la factibilidad de su replicación en otros contextos educacionales. Es importante considerar que esta evaluación de impacto considera solo las actividades realizadas en su primer año de ejecución, y el posible efecto de los restantes dos años del programa será evaluado al finalizar el proceso.

La agenda de investigación en educación para el emprendimiento o de generación de espíritu emprendedor debe incorporar mediciones de impacto de este tipo y avanzar sobre el estudio de la factibilidad de generación de otras capacidades que son significativas en una sociedad en donde la innovación y el emprendimiento se han tornado valores. 
URL: http://www.una.ac.cr/educare

CORREO: educare@una.cr

\section{Referencias}

Artola, T. y Barraca, J. (2004). Creatividad e imaginación. Un nuevo instrumento de medida: La PIC. eduPsykhé, 3(1), 73-93.

Charney, A. y Libecap, G. (2000). Impact of Entrepreneurship Education [Impacto de la Educación para el Emprendimiento]. Kansas: Kauffman Center for Entrepreneurial Leadership.

Comisión Europea. (2006). Entrepreneurship Education in Europe: Fostering Entrepreneurial Mindsets Through Education and Learning [Educación para el emprendimiento en Europa: Fomentando la mentalidad emprendedora a través de la educación y el aprendizaje]. Oslo: Autor.

Cox, C. (Ed.). (2005). Políticas educacionales en el cambio de siglo: La reforma escolar en Chile (2ª ed.). Santiago, Chile: Editorial Universitaria.

Drucker, P. (1993). Innovation and Entrepreneurship [Educación y emprendimiento]. Nueva York: Harper Collins Publishers.

Guilford, J. P. (1967). Creativity: Yesterday, Today and Tomorrow [Creatividad: Ayer, hoy y mañana]. The Journal of Creative Behavior, 1(1), 3-14. doi:1 0.1002/j.2162-6057.1967.tb00002

Kuratko, D. F. (setiembre, 2005). The Emergence of Entrepreneurship Education: Development, Trends, and Challenges [La emergencia de la educación para el emprendimiento: Desarrollo, tendencias y cambios]. Entrepreneurship Theory and Practice, 29(5), 577-598. doi: 10.1111/j.1540-6520.2005.00099.x

Mueller, S. (2011). Increasing Entrepreneurial Intention: Effective Entrepreneurship Course Characteristics [Incrementando la intención de emprender: Características de un curso de emprendimiento efectivo]. International Journal of Entrepreneurship and Small Business, 13(1), 55-74. doi: 10.1504/JJESB.2011.040416

Oosterbeek, H., van Praag, M. y ljsselstein, A. (2010). The Impact of Entrepreneurship Education on Entrepreneurship Skills and Motivation [El impacto de la educación para el emprendimiento en habilidades y motivación para el emprendimiento]. European Economic Review, 54(3), 442-454. doi: 10.1016/j.euroecorev.2009.08.002

Peterman, N. E. y Kennedy, J. (diciembre, 2003). Enterprise Education: Influencing Students' Perceptions of Entrepreneurship [Educación de empresa: Influenciando la percepción del estudiantado sobre el emprendimiento]. Entrepreneurship Theory and Practice, 28(2), 129144. doi: 10.1046/j.1540-6520.2003.00035.x

Romaní, G., Didonet, S., Contuliano, S.-H. y Portilla, R. (2013). Propensity of University Students in the Region of Antofagasta, Chile to Create Enterprise [Propensión de estudiantes universitarios en la región de Antofagasta, Chile para la creación de empresas]. Journal of Education for Business, 88(5), 253-264. doi: 10.1080/08832323.2012.690353 
Rosenbaum, P. R. (2002). Observational Studies [Estudios observacionales] (2 ${ }^{\mathrm{a}}$ ed.). Nueva York: Springer.

Rosenbaum, P. R. y Rubin, D. B. (1983). The Central Role of the Propensity Score in Observational Studies for Causal Effects [El rol central del propensity score en estudios observacionales para efectos causales]. Biometrika, 70(1), 41-55. doi: 10.1093/biomet/70.1.41

Rosendahl, L., Sloof, R. y Van Praag, M. (2012). The Effect of Early Entrepreneurship Education:Evidence from a Randomized Field Experiment [El efecto de la educación para el emprendimiento temprano: Evidencia desde un experimento aleatorio] (SSRN Scholarly Paper No. ID 2044735). Rochester, NY: Social Science Research Network.

Salazar, J., Husted, B. W. y Biehl, M. (enero, 2012). Thoughts on the Evaluation of Corporate Social Performance Through Projects [Reflexiones en la evaluación del desempeño social corporativo a través de proyectos]. Journal of Business Ethics, 105(2), 175-186. doi: 10.1007/s10551-011-0957-z

Schumpeter, J. A. (1971). Capitalismo, socialismo y democracia. Madrid: Aguilar.

Souitaris, V., Zerbinati, S. y Al-Laham, A. (julio, 2007). Do Entrepreneurship Programmes Raise Entrepreneurial Intention of Science and Engineering Students? The Effect of Learning, Inspiration and Resources [¿Los programas de emprendedores aumentan la intención emprendedora de estudiantes de ciencias e ingeniería: El efecto de aprendizaje, inspiración y recursos]. Journal of Business Venturing, 22(4), 566-591. doi: 10.1016/j.jbusvent.2006.05.002

Torche, F. (mayo, 2010). Educational Assortative Mating and Economic Inequality: A Comparative Analysis of Three Latin American Countries [Emparejamiento educacional selectivo e inequidad económica: Un análisis comparado de tres países latinoamericanos]. Demography, 47(2), 481-502. doi: 10.1353/dem.0.0109

Varela, R. y Jiménez, J. (2001). The Effect of Entrepreneurship Education in the Universities of Cali [El efecto de la educación para el emprendimiento en las universidades de Cali]. Presentado en Frontiers of Entrepreneurship Research, Babson Conference, Jönköpink, Suecia.

Von Graevenitz, G., Harhoff, D. y Weber, R. (2010). The Effects of Entrepreneurship Education [EI efecto de la educación para el emprendimiento]. Journal of Behavior \& Organization, 76(1), 90-112. doi: 10.1016/j.jebo.2010.02.015 


\section{URL: http://www.una.ac.cr/educare}

CORREO: educare@una.cr

\section{Material complementario}

Talleres de Etapa I "Comprender el emprender"

\begin{tabular}{|c|c|c|c|c|c|c|c|c|c|}
\hline Taller & Descripción & 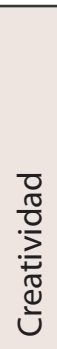 & 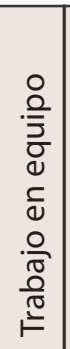 & 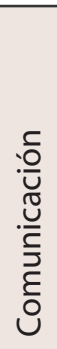 & $\begin{array}{l}\text { O } \\
\text { N } \\
\frac{0}{2} \\
\frac{0}{-}\end{array}$ & 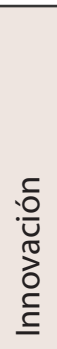 & 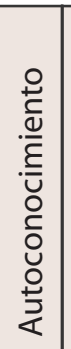 & 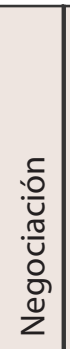 & 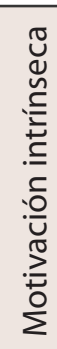 \\
\hline $\begin{array}{l}\text { La caja no } \\
\text { es caja }\end{array}$ & $\begin{array}{l}\text { Actividad de pensamiento de diseño, donde diversos } \\
\text { equipos deben generar, desde un estímulo concreto } \\
\text { (una caja), el desarrollo de un nuevo prototipo de } \\
\text { producto, a través del uso del proceso de generación } \\
\text { de ideas creativas. }\end{array}$ & $x$ & $x$ & $x$ & $x$ & $x$ & & $x$ & $x$ \\
\hline \begin{tabular}{l|} 
Formación \\
del concepto \\
"creatividad"
\end{tabular} & $\begin{array}{l}\text { Actividad de generación de ideas, donde desde } \\
\text { conceptos únicos los participantes deben construir } \\
\text { definiciones integrales, partiendo desde sus } \\
\text { conocimientos propios asociados a la temática de la } \\
\text { creatividad. }\end{array}$ & $x$ & & $x$ & & $x$ & $x$ & & $x$ \\
\hline $\begin{array}{l}\text { Notas que } \\
\text { generan } \\
\text { ideas }\end{array}$ & $\begin{array}{l}\text { Actividad de pensamiento de diseño, donde mediante } \\
\text { un trabajo individual, los participantes deben generar } \\
\text { desde un estímulo abstracto un producto creativo, } \\
\text { utilizando procesos imaginativos de desarrollo. }\end{array}$ & $x$ & & $x$ & & $x$ & $x$ & & $x$ \\
\hline Yo letra & $\begin{array}{l}\text { Actividad de diseño, donde los participantes deben a } \\
\text { través de un trabajo colaborativo formar palabras, en } \\
\text { base a un proceso de planificación y organización previa. }\end{array}$ & $x$ & $x$ & $x$ & & $x$ & & & \\
\hline Cranéate & \begin{tabular}{|l|} 
Actividad formato competencia, donde una serie \\
de equipos deben resolver una serie de problemas \\
de lógica o de pensamiento divergente, donde se \\
presentan una cadena de obstáculos a resolver \\
mediante la creación de un dispositivo para procesar \\
las preguntas entregadas
\end{tabular} & $x$ & $x$ & $x$ & $x$ & & & $x$ & $x$ \\
\hline
\end{tabular}

\section{Cómo citar este artículo en APA:}

Olivos, F., Álvarez, I. y Díaz, F. (2013). Impacto de la educación para el emprendimiento en la creatividad: Una experiencia en Chile con Propensity Score Matching. Revista Electrónica Educare, 17(3), 259-276. Recuperado de http://www.revistas.una.ac.cr/index.php/EDUCARE/issue/current

Nota: Para citar este artículo en otros sistemas puede consultar el hipervínculo "Como citar el artículo" en la barra derecha de nuestro sitio web:

http://www.revistas.una.ac.cr/index.php/EDUCARE/index 